\title{
Detection of Leptospira kmetyi at recreational areas in Peninsular Malaysia
}

\begin{abstract}
Humans can be infected by pathogenic Leptospira through contact with infected animals or contaminated environments. Recreational exposure has been associated with human leptospirosis; however, there is a lack of information on the distribution of Leptospira spp. in recreational areas. Thus, we conducted this study to detect and describe the distributions of Leptospira spp. and to determine the prevalence of pathogenic leptospires at recreational areas in Peninsular Malaysia. Soil and water samples were randomly collected from 33 recreational areas in Peninsular Malaysia from December 2018 to April 2019. Each culture was examined under dark-field microscopy prior to genus confirmation by polymerase chain reaction using primers for the 16S rRNA gene. A total of 390 water and soil samples were collected, and 131 cultures were positive for Leptospira under dark-field microscope examination. Leptospira was identified in most of the recreational areas sampled, and at least one pathogenic Leptospira species was isolated from 17 recreational areas. The prevalence of saprophytic, intermediate, and pathogenic Leptospira spp. was $19.7 \%, 5.6 \%$, and $8.2 \%$, respectively. The dominant pathogenic species found in the samples was Leptospira kmetyi. This study provides important data on the distribution and prevalence of Leptospira spp. from soil and water, as well as the dominant pathogenic species, at recreational areas in Peninsular Malaysia. Therefore, preventive measures should be taken to ensure the safety of visitors.
\end{abstract}

Keyword: 16S rRNA; Leptospira; Polymerase chain reaction; Prevalence; Recreational area 